ISSN 1561-2430 (Print)

ISSN 2524-2415 (Online)

УДК 519.216.73

https://doi.org/10.29235/1561-2430-2020-56-1-36-50

Поступила в редакцию 22.10.2019

Received 22.10.2019

\title{
М. М. Васьковский
}

Белорусский государственный университет, Минск, Беларусь

\section{СТОХАСТИЧЕСКИЕ ДИФФЕРЕНЦИАЛЬНЫЕ УРАВНЕНИЯ СМЕШАННОГО ТИПА СО СТАНДАРТНЫМИ И ДРОБНЫМИ БРОУНОВСКИМИ ДВИЖЕНИЯМИ С ИНДЕКСАМИ ХЕРСТА, БОЛЬШИМИ 1/3}

\begin{abstract}
Аннотация. Для стохастических дифференциальных уравнений смешанного типа, управляемых стандартными и дробными броуновскими движениями с индексами Херста, большими $1 / 3$, доказаны теоремы о существовании, единственности и непрерывной зависимости решений от начальных данных. Для таких уравнений получен аналог формулы Ито замены переменных. Найдены асимптотические разложения функционалов от решений стохастических дифференциальных уравнений смешанного типа при малых значениях времени. В коммутативном случае получены аналоги дифференциальных уравнений Колмогорова для математических ожиданий и плотностей распределений решений. Рассматривается приложение стохастических дифференциальных уравнений смешанного типа к решению проблемы экстраполяции макроэкономических факторов при моделировании кредитных рисков.

Ключевые слова: дробное броуновское движение, формула Ито, стохастическое дифференциальное уравнение, интеграл Ито, интеграл Губинелли

Для цитирования. Васьковский, М. М. Стохастические дифференциальные уравнения смешанного типа со стандартными и дробными броуновскими движениями с индексами Херста, большими 1/3 / М. М. Васьковский // Вес. Нац. акад. навук Беларусі. Сер. фіз.-мат. навук. - 2020. - Т. 56, № 1. - С. 36-50. https://doi.org/10.29235/1561-24302020-56-1-36-50
\end{abstract}

\author{
Maksim M. Vas'kovskii \\ Belarusian State University, Minsk, Belarus
}

\begin{abstract}
MIXED-TYPE STOCHASTIC DIFFERENTIAL EQUATIONS DRIVEN BY STANDARD AND FRACTIONAL BROWNIAN MOTIONS WITH HURST INDICES GREATER THAN 1/3
\end{abstract}

\begin{abstract}
In this paper we consider mixed-type stochastic differential equations driven by standard and fractional Brownian motions with Hurst indices greater than 1/3. There are proved theorems on the existence, uniqueness, and continuous dependence of solutions on the initial data. We provide an analog of the Ito formula to change variables. Asymptotic expansions of functionals on the solutions of mixed-type stochastic differential equations for small times are obtained. We receive analogs of the Kolmogorov equations for mathematical expectations and probability densities in the commutative case. Finally, we consider an application of mixed-type stochastic differential equations to solving the problem of macroeconomic variables extrapolation in credit risks models.

Keywords: fractional Brownian motion, Ito formula, stochastic differential equation, Ito integral, Gubinelli integral

For citation. Vas'kovskii M. M. Mixed-type stochastic differential equations driven by standard and fractional Brownian motions with Hurst indices greater than 1/3. Vestsi Natsyianal'nai akademii navuk Belarusi. Seryia fizika-matematychnykh navuk $=$ Proceedings of the National Academy of Sciences of Belarus. Physics and Mathematics series, 2020, vol. 56, no. 1, pp. 36-50 (in Russian). https://doi.org/10.29235/1561-2430-2020-56-1-36-50
\end{abstract}

Стохастические дифференциальные уравнения, управляемые стандартными и дробными броуновскими движениями, используются в финансовой математике и задачах фильтрации [1-5]. В статье [6] рассматривались стохастические дифференциальные уравнения Стратоновича, управляемые дробными броуновскими движениями с различными индексами Херста, большими 1/3. Но для многих приложений важную роль играют так называемые уравнения смешанного типа [4], в которых интегралы по стандартному броуновскому движению понимаются как интегралы Ито, а интегралы по дробным броуновским движениям - как потраекторные интегралы. Смешанные уравнения, содержащие дробные броуновские движения с индексами Херста, большими 1/2, изучались в работах [7-18]. В настоящей статье исследуются стохастиче-

(C) Васьковский М. М., 2020 
ские дифференциальные уравнения смешанного типа, управляемые стандартными и дробными броуновскими движениями с индексами Херста, большими 1/3. Для таких уравнений интегралы по дробным броуновским движениям не могут быть определены как потраекторные интегралы Янга. Следуя теории грубых траекторий $[19,20]$, мы определяем интегралы по дробным броуновским движениям как потраекторные интегралы Губинелли. Развивая идеи работы [6], в настоящей статье для упомянутого класса стохастических дифференциальных уравнений доказываются теоремы о существовании, единственности и непрерывной зависимости решений от начальных данных. Выводится формула замены переменных, с помощью которой исследуются асимптотические свойства функционалов от решений при малых значениях времени. В статье получены аналоги уравнений Колмогорова для математических ожиданий и плотностей распределений решений. В заключение рассматривается приложение стохастических дифференциальных уравнений смешанного типа к решению проблемы экстраполяции макроэкономических факторов при моделировании кредитных рисков.

Пусть на вероятностном пространстве $(\Omega, \mathcal{F}, P)$ заданы независимые $d_{1}$-мерное дробное броуновское движение $B_{t}=\left(B_{t}^{(1)}, \ldots, B_{t}^{\left(d_{1}\right)}\right)$ с показателями Херста $H_{1}, \ldots, H_{d_{1}} \in\left(\frac{1}{3}, \frac{1}{2}\right) \cup\left(\frac{1}{2}, 1\right)$ и $d_{2}$-мерное стандартное броуновское движение $W_{t}=\left(W_{t}^{(1)}, \ldots, W_{t}^{\left(d_{2}\right)}\right)$.

Рассмотрим стохастическое дифференциальное уравнение

$$
d X_{t}=b\left(X_{t}\right) d t+\sigma\left(X_{t}\right) d B_{t}+h\left(X_{t}\right) d W(t), \quad t \in[0, T],
$$

где $b: \mathbb{R}^{n} \rightarrow \mathbb{R}^{n}, \quad \sigma: \mathbb{R}^{n} \rightarrow \mathbb{R}^{n \times d_{1}}, h: \mathbb{R}^{n} \rightarrow \mathbb{R}^{n \times d_{2}}$ - детерминированные функции.

Определим функцию $f: \mathbb{R}^{n} \rightarrow \mathbb{R}^{n \times(d+1)}, f=\left(f_{0}, f_{1}, \ldots, f_{d}\right)$, где $f_{1}, \ldots, f_{d}-$ компоненты функций $\sigma$ и $h$ соответственно, $f_{0}=b, d=d_{1}=d_{2}$. Случайный процесс $B_{t}^{H}=\left(t, B_{t}, W_{t}\right)$ также будем называть дробным броуновским движением с многомерным индексом Херста $H=\left(H_{0}, \ldots, H_{d}\right)$, где $H_{0}=1, H_{i}=1 / 2$ при $d_{1}<i<d$. Уравнение (1) может быть записано следующим образом:

$$
d X_{t}=f\left(X_{t}\right) d B_{t}^{H}, \quad t \in[0, T] .
$$

В дальнейшем будем опускать индекс $H$ у процесса $B_{t}^{H}$, а через $H$ будем обозначать некоторое фиксированное действительное число из интервала $H \in\left(1 / 3, H_{\min }\right)$, где $H_{\min }$ - минимальное из значений $H_{i}, i=0, \ldots, d$.

Пусть $V$ - некоторое конечномерное евклидово пространство, $\alpha \in\left(\frac{1}{3}, \frac{1}{2}\right]$. Множество функций $z:[0, T] \rightarrow V$, непрерывных по Гельдеру с показателем $\alpha$, для которых

$$
|z|_{\alpha}:=\sup _{s, t \in[0, T], s \neq t} \frac{\|z(t)-z(s)\|_{V}}{|t-s|^{\alpha}}<\infty,
$$

обозначим через $C^{\alpha}([0, T], V)$.

Пусть $W$ - конечномерное евклидово пространство, $\mathcal{L}(W, V)$ - пространство ограниченных линейных операторов, действующих из $W$ в $V$. Множество функций $y:[0, T] \times[0, T] \rightarrow \mathcal{L}(W, V)$, для которых

$$
|y|_{2 \alpha}:=\sup _{s, t \in[0, T], s \neq t} \frac{\|y(t, s)\|_{\mathcal{L}(W, V)}}{|t-s|^{2 \alpha}}<\infty,
$$

обозначим через $C_{2}^{2 \alpha}\left([0, T]^{2}, \mathcal{L}(W, V)\right)$.

Говорят, что отображение $\mathbb{Y}:[0, T]^{2} \rightarrow V \otimes V$ является процессом второго порядка над функцией $Y:[0, T] \rightarrow V$, если оно удовлетворяет следующему тождеству Чена: 


$$
\mathbb{Y}(s, t)-\mathbb{Y}(s, u)-\mathbb{Y}(u, t)=(Y(u)-Y(s)) \otimes(Y(t)-Y(u))
$$

для любой тройки $(s, u, t) \in[0, T]^{3}$.

Через $\mathcal{C}^{\alpha}([0, T], V)$ обозначим множество $\alpha$-непрерывных по Гельдеру грубых траекторий над $V$, т. е. множество всех пар $(Y, \mathbb{Y})$ таких, что функция $Y$ принадлежит $C^{\alpha}([0, T], V)$, а $\mathbb{Y} \in C_{2}^{2 \alpha}\left([0, T]^{2}, V \otimes V\right)$ является процессом второго порядка над $Y$.

Под множеством $\alpha$-непрерывных по Гельдеру геометрических грубых траекторий над $V$ понимают множество всех пар $(Y, \mathbb{Y}) \in \mathcal{C}^{\alpha}([0, T], V)$, для которых имеет место следующее соотношение:

$$
\operatorname{Sym}(\mathbb{Y}(s, t)):=\frac{1}{2}\left(\mathbb{Y}(s, t)+\mathbb{Y}^{\mathrm{T}}(s, t)\right)=\frac{1}{2} Y(s, t) \otimes Y(s, t)
$$

для любой пары $(s, t) \in[0, T]^{2}$.

Говорят, что функция $Y \in C^{\alpha}([0, T], \mathcal{L}(W, V))$ управляется функцией $Z \in C^{\alpha}([0, T], W)$, если существует элемент $Y^{\prime} \in C^{\alpha}([0, T], \mathcal{L}(W, \mathcal{L}(W, V)))$ (называемый производной Губинелли $Y$ ), такой что выражение $R^{Y}(s, t)=Y(s, t)-Y_{s}^{\prime} Z(s, t)$ удовлетворяет неравенству $\left|R^{Y}\right|_{2 \alpha}<+\infty$. Множество всех $\left(Y, Y^{\prime}\right)$ таких, что $Y$ управляется функцией $Z$, будем обозначать $\mathcal{D}_{Z}^{2 \alpha}([0, T], \mathcal{L}(W, V))$. Множество $\mathcal{D}_{Z}^{2 \alpha}([0, T], \mathcal{L}(W, V))$ является банаховым пространством с нормой

$$
\left\|\left(Y, Y^{\prime}\right)\right\|=\|Y(0)\|+\left\|Y^{\prime}(0)\right\|+\left|Y^{\prime}\right|_{\alpha}+\left|R^{Y}\right|_{2 \alpha} .
$$

Пусть $(Z, \mathbb{Z}) \in \mathcal{C}^{\alpha}([0, T], W)$, а также $\left(Y, Y^{\prime}\right) \in \mathcal{D}_{Z}^{2 \alpha}([0, T], \mathcal{L}(W, V))$. Потраекторным интегралом Губинелли от $Y$ по отображению $Z$ называют предел интегральных сумм

$$
\int_{0}^{T} Y d Z=\lim _{|\mathcal{P}| \rightarrow 0} \sum_{t_{i}, t_{i+1} \in \mathcal{P}}\left(Y\left(t_{i}\right) Z\left(t_{i}, t_{i+1}\right)+Y^{\prime}\left(t_{i}\right) \mathbb{Z}\left(t_{i}, t_{i+1}\right)\right),
$$

где $|\mathcal{P}|=\max \left|t_{i+1}-t_{i}\right|-$ диаметр разбиения $\mathcal{P}=\left\{0=t_{0}<t_{1}<\ldots<t_{l}=T\right\}$, а предел понимается не зависящим от последовательности разбиений $\mathcal{P}$. Если $Z \in C^{\beta}([0, T], W), \quad Y \in C^{\gamma}([0, T], \mathcal{L}(W, V))$, $\beta+\gamma>1$, то потраекторный интеграл Губинелли совпадает с потраекторным интегралом Янга, который может быть определен как предел интегральных сумм

$$
\int_{0}^{T} Y d Z=\lim _{|\mathcal{P}| \rightarrow 0} \sum_{t_{i}, t_{i+1} \in \mathcal{P}} Y\left(t_{i}\right) Z\left(t_{i}, t_{i+1}\right) .
$$

Определим процесс второго порядка $\mathbb{B}:[0, T]^{2} \times \Omega \rightarrow \mathbb{R}^{(d+1) \times(d+1)}$ над дробным броуновским движением $B$ следующим образом:

$$
\begin{gathered}
\mathbb{B}_{s, t}=\left(\mathbb{B}_{s, t}^{(i, j)}\right)_{i, j=0}^{d}, \\
\mathbb{B}_{s, t}^{(i, j)}=\lim _{|\mathcal{P}| \rightarrow 0} \int_{\mathcal{P}} B_{s, r}^{(i)} d B_{r}^{(j)}, \int_{\mathcal{P}} B_{s, r}^{(i)} d B_{r}^{(j)}=\sum_{t_{k}, t_{k+1} \in \mathcal{P}} B_{s, t_{k}}^{(i)} B_{t_{k}, t_{k+1}}^{(j)}, \quad 1 \leq i<j \leq d, \\
\mathbb{B}_{s, t}^{(0, j)}=\int_{s}^{t} B_{s, r}^{(j)} d r=\lim _{|\mathcal{P}| \rightarrow 0} \sum_{t_{k}, t_{k+1} \in \mathcal{P}} B_{s, t_{k}}^{(j)}\left(t_{k+1}-t_{k}\right), \quad 1 \leq j \leq d, \\
\mathbb{B}_{s, t}^{(i, i)}=\frac{1}{2}\left(B_{s, t}^{(i)}\right)^{2}-\frac{1}{2}(t-s) 1_{\left\{H_{i}=1 / 2\right\}}, \quad 0 \leq i \leq d, \\
\mathbb{B}_{s, t}^{(i, j)}=-\mathbb{B}_{s, t}^{(j, i)}+B_{s, t}^{(i)} B_{s, t}^{(j)}, \quad 0 \leq j<i \leq d,
\end{gathered}
$$

для любой пары $(s, t) \in[0, T]^{2}$, где $1_{\left\{H_{i}=1 / 2\right\}}=1$, если $H_{i}=1 / 2,1_{\left\{H_{i}=1 / 2\right\}}=0$, если $H_{i} \neq 1 / 2$, $\mathcal{P}=\left\{s=t_{0}<t_{1}<\ldots<t_{l}=t\right\}-$ произвольное разбиение отрезка $[s, t],|\mathcal{P}|=\max \left|t_{k+1}-t_{k}\right|$, а все пределы понимаются не зависящими от последовательности разбиений $\mathcal{P}$. Здесь обозначения 
$L^{2}$ П.н.

$=,=$ применяются для того, чтобы показать, что соответствующие пределы понимаются в смысле $L^{2}(\Omega, \mathcal{F}, P)$ и $P=1$ соответственно.

Замеч чн ие 1 . При $1 \leq i<j \leq d$ процессы $\mathbb{B}_{s, t}^{(i, j)}$ являются интегралами Ито, которые совпадают с интегралами Стратоновича в силу независимости процессов $B^{(i)}$ и $B^{(j)}$ (см. [21, разд. 5.2]). В свою очередь процесс $\mathbb{B}^{(i, i)}$ соответствует интегралу Стратоновича $\int_{s}^{t} B_{s, r}^{(i)} d B_{r}^{(i)}$ при $H_{i} \neq 1 / 2$ и интегралу Ито при $H_{i}=1 / 2$. Наконец формула $\mathbb{B}_{s, t}^{(i, j)}=-\mathbb{B}_{s, t}^{(j, i)}+B_{s, t}^{(i)} B_{s, t}^{(j)}$ является формулой интегрирования по частям для интегралов Стратоновича.

3 амечани е 2. Нетрудно видеть, что

$$
\operatorname{Sym}\left(\mathbb{B}_{s, t}+\frac{1}{2}(t-s) I_{\bar{H}}\right)=\frac{1}{2} B_{s, t} \otimes B_{s, t},
$$

где

$$
I_{\bar{H}}=I_{\left(H_{0}, \ldots, H_{d}\right)}=\operatorname{diag}\left(1_{\left\{H_{0}=1 / 2\right\}}, \ldots, 1_{\left\{H_{d}=1 / 2\right\}}\right) .
$$

Пре дложен ие 1 [6]. Для любого фиксированного $H \in(1 / 3,1 / 2]$ такого, что $H<H_{\min }=$ $=\min H_{i}$ имеет место включение $(B, \mathbb{B}) \in \mathcal{C}^{H}\left([0, T], \mathbb{R}^{d+1}\right) n$. н., и, более того, $\mathbb{E}\|\mathbb{B}\|_{2 H}^{q}<\infty$ для $i=0, \ldots, d$ любого $q \geq 1$.

О пр еделен и е 1 . Случайный процесс $X_{t}$ такой, что $\left(X, X^{\prime}\right) \in \mathcal{D}_{B}^{2 H}\left([0, T], \mathbb{R}^{n}\right)$ п. н., будем называть решением уравнения (2), если он п. н. удовлетворяет равенству

$$
X_{t}=X_{0}+\int_{0}^{t} f\left(X_{s}\right) d B_{s}, \quad t \in[0, T],
$$

где интеграл понимается как потраекторный интеграл Губинелли. Пусть $x \in \mathbb{R}^{n}$. Решение уравнения (2) с начальным условием $X_{0}=x$ будем называть п. н. единственным, если для любого решения $Y_{t}$ уравнения (2) с начальным условием $Y_{0}=x$ выполняется равенство $P\left(X_{t}=Y_{t} \quad \forall t \in[0, T]\right)=1$.

Те орема 1. Если $f \in C_{b}^{3}\left(\mathbb{R}^{n}, \mathbb{R}^{n \times(d+1)}\right)$, то для любого $x \in \mathbb{R}^{n}$ уравнение (2) имеет единственное решение с начальнылм условием $X_{0}=x$, причем $X^{\prime}=f(X),\left(f(X),(f(X))^{\prime}\right) \in$ $\in \mathcal{D}_{B}^{2 H}\left([0, T], \mathbb{R}^{n \times(d+1)}\right)$ n. $H$.

Доказательство существования и единственности функции $X(t, \omega)$, удовлетворящей почти наверное интегральному уравнению (3), вытекает из теоремы 3.13 в [22]. Измеримость процесса $X(t, \omega)$ следует из непрерывности отображения Ито - Лайонса, установленной в утверждении 2 теоремы 3.13 в [22], и сходимости диадных аппроксимаций $B_{t}(m)$ к дробному броуновскому движению $B_{t}$, доказанной в теореме 2 в [23].

Наряду с уравнением (2) рассмотрим аналогичное уравнение с возмущенной правой частью

$$
d \widetilde{X}_{t}=\widetilde{f}\left(\widetilde{X}_{t}\right) d B_{t}, \quad t \in[0, T]
$$

где $\widetilde{f}-(n \times(d+1))$-матрица, столбцами которой являются векторы $\widetilde{f}_{i}: \mathbb{R}^{n} \rightarrow \mathbb{R}^{n}, i=0, \ldots, d$.

Из предложения 1 и доказательства теорем 7.3 и 7.4 [18] вытекает теорема о непрерывной зависимости решений уравнения (2) от начальных условий и правых частей.

Те о рем а 2. Пусть $f, \widetilde{f} \in C_{b}^{4}\left(\mathbb{R}^{n}, \mathbb{R}^{n \times(d+1)}\right)$, причем функция $\widetilde{f}$ такова, что $\|f-\widetilde{f}\|_{C_{b}^{2}} \leq 1$. Обозначим через $X_{t}, \widetilde{X}_{t}$ решения уравнений (2), (4) с начальныли условиями $X_{0}=\xi, \widetilde{X}_{0}=\tilde{\xi}$ соответственно. Тогда:

1) почти наверное справедлива следующая оценка:

$$
\|X-\widetilde{X}\|_{H} \leq C\left(|\xi-\tilde{\xi}|+\|f-\widetilde{f}\|_{C_{b}^{2}}\right)
$$


для некоторой случайной величины

$$
C=C\left(H, T,\|f\|_{C_{b}^{4}},\|B\|_{H},\|\mathbb{B}\|_{2 H}\right)
$$

2) имеет место следующее неравенство:

$$
\mathbb{E}\left(\ln \|X-\widetilde{X}\|_{H}\right) \leq C+\ln \left(\mathbb{E}|\xi-\tilde{\xi}|+\|f-\widetilde{f}\|_{C_{b}^{2}}\right),
$$

где $C=C\left(H, H_{1}, \ldots, H_{d}, T,\|f\|_{C_{b}^{4}}\right)$ - некоторая постоянная.

Следующая теорема является обобщением известной формулы Ито о замене переменных в дифферециальных выражениях на класс уравнений (2).

Те о рема 3. Пусть $f \in C_{b}^{3}\left(\mathbb{R}^{n}, \mathbb{R}^{n \times(d+1)}\right), g \in C_{b}^{3}\left(\mathbb{R}^{n}, \mathbb{R}\right)$. Тогда для любых $s, t \in[0, T] n$. н. справедлива следующая формула:

$$
g\left(X_{t}\right)=g\left(X_{s}\right)+\int_{s}^{t} D g\left(X_{r}\right) f\left(X_{r}\right) d B_{r}+\int_{s}^{t} \frac{1}{2} \operatorname{tr}\left(\left(f\left(X_{r}\right) I_{\bar{H}}\right)^{T} D^{2} g\left(X_{r}\right)\left(f\left(X_{r}\right) I_{\bar{H}}\right)\right) d r, \quad s, t \in[0, T],
$$

где $X_{t}$ - решение уравнения (2).

Дока з ат ел ь с т о. Зафиксируем произвольные $s, t \in[0, T], s \leq t$, и рассмотрим разбиение отрезка $[s, t]$ точками

$$
\mathcal{P}^{(N)}=\left\{s=t_{0}<t_{1}<\ldots<t_{N}=t\right\}, \quad\left|\mathcal{P}^{(N)}\right|=\max _{i=0, \ldots, N-1}\left|t_{i+1}-t_{i}\right| .
$$

Будем обозначать $X^{\otimes m}=\underbrace{X \otimes \ldots \otimes X}_{m}$. Все равенства и неравенства ниже для случайных величин будем понимать выполненными почти наверное. Из формулы Тейлора вытекает справедливость соотношения

$$
\begin{gathered}
g\left(X_{t}\right)-g\left(X_{s}\right)=\sum_{i=0}^{N-1}\left(g\left(X_{t_{i+1}}\right)-g\left(X_{t_{i}}\right)\right)= \\
=\sum_{i=0}^{N-1}\left(D g\left(X_{t_{i}}\right) X_{t_{i}, t_{i+1}}+\frac{1}{2} D^{2} g\left(X_{t_{i}}\right) X_{t_{i}, t_{i+1}}^{\otimes 2}+\frac{1}{6} D^{3} g\left(X_{t_{i}}+\theta_{i} X_{t_{i}, t_{i+1}}\right) X_{t_{i}, t_{i+1}}^{\otimes 3}\right),
\end{gathered}
$$

для некоторых $\theta_{i} \in(0,1)$.

Оценим последнее слагаемое в сумме (5). Поскольку $X \in C^{H}\left([0, T], \mathbb{R}^{n}\right)$ и $3 H>1$, то имеем

$$
\begin{gathered}
\left|\sum_{i=0}^{N-1} \frac{1}{6} D^{3} g\left(X_{t_{i}}+\theta_{i} X_{t_{i}, t_{i+1}}\right) X_{t_{i}, t_{i+1}}^{\otimes 3}\right| \leq \frac{1}{6}\left\|D^{3} g\right\|_{\infty}\|X\|_{H}^{3} \sum_{i=0}^{N-1}\left|t_{i+1}-t_{i}\right|^{3 H}= \\
=\frac{1}{6}\left\|D^{3} g\right\|_{\infty}\|X\|_{H}^{3}(t-s)\left|\mathcal{P}^{(N)}\right|^{3 H-1}=O\left(\left|\mathcal{P}^{(N)}\right|^{3 H-1}\right) .
\end{gathered}
$$

Из теоремы 4.10 [1] следует, что

$$
X_{t_{i}, t_{i+1}}=\int_{t_{i}}^{t_{i+1}} f\left(X_{r}\right) d B_{r}=f\left(X_{t_{i}}\right) B_{t_{i}, t_{i+1}}+D f\left(X_{t_{i}}\right) f\left(X_{t_{i}}\right) \mathbb{B}_{t_{i}, t_{i+1}}+O\left(\left|t_{i+1}-t_{i}\right|^{3 H}\right),
$$

причем константа в $O\left(\left|t_{i+1}-t_{i}\right|^{3 H}\right)$ зависит только от $f, B$ и $X$ и не зависит от разбиения $\mathcal{P}^{(N)}$. Учитывая неравенства

$$
\left|f\left(X_{t_{i}}\right) B_{t_{i}, t_{i+1}}\right| \leq\|f\|_{\infty}\|B\|_{H}\left|t_{i+1}-t_{i}\right|^{H}
$$




$$
\left|D f\left(X_{t_{i}}\right) f\left(X_{t_{i}}\right) \mathbb{B}_{t_{i}, t_{i+1}}\right| \leq\|f\|_{C_{b}^{2}}^{2}\|\mathbb{B}\|_{2 H}\left|t_{i+1}-t_{i}\right|^{2 H},
$$

и умножая соотношение (7) тензорно на себя, получим соотношения

$$
\begin{gathered}
X_{t_{i}, t_{i+1}}^{\otimes 2}=\left(f\left(X_{t_{i}}\right) B_{t_{i}, t_{i+1}}\right)^{\otimes 2}+\left(D f\left(X_{t_{i}}\right) f\left(X_{t_{i}}\right) \mathbb{B}_{t_{i}, t_{i+1}}\right)^{\otimes 2}+ \\
+f\left(X_{t_{i}}\right) B_{t_{i}, t_{i+1}} \otimes D f\left(X_{t_{i}}\right) f\left(X_{t_{i}}\right) \mathbb{B}_{t_{i}, t_{i+1}}+ \\
+D f\left(X_{t_{i}}\right) f\left(X_{t_{i}}\right) \mathbb{B}_{t_{i}, t_{i+1}} \otimes f\left(X_{t_{i}}\right) B_{t_{i}, t_{i+1}}+O\left(\left|t_{i+1}-t_{i}\right|^{4 H}\right)= \\
=\left(f\left(X_{t_{i}}\right) B_{t_{i}, t_{i+1}}\right)^{\otimes 2}+O\left(\left|t_{i+1}-t_{i}\right|^{3 H}\right) .
\end{gathered}
$$

Подставляя (6)-(8) в (5) и замечая, что

$$
\begin{gathered}
D^{2} g\left(X_{t_{i}}\right)\left(f\left(X_{t_{i}}\right) B_{t_{i}, t_{i+1}}\right)^{\otimes 2}=\left(f\left(X_{t_{i}}\right) B_{t_{i}, t_{i+1}}\right)^{\mathrm{T}} D^{2} g\left(X_{t_{i}}\right)\left(f\left(X_{t_{i}}\right) B_{t_{i}, t_{i+1}}\right)= \\
=\left(B_{t_{i}, t_{i+1}}\right)^{\mathrm{T}}\left(f\left(X_{t_{i}}\right)^{\mathrm{T}} D^{2} g\left(X_{t_{i}}\right) f\left(X_{t_{i}}\right)\right) B_{t_{i}, t_{i+1}}= \\
=\left(f\left(X_{t_{i}}\right)^{\mathrm{T}} D^{2} g\left(X_{t_{i}}\right) f\left(X_{t_{i}}\right)\right)\left(B_{t_{i}, t_{i+1}}\right)^{\otimes 2}
\end{gathered}
$$

получим соотношения

$$
\begin{gathered}
g\left(X_{t}\right)-g\left(X_{s}\right)=\sum_{i=0}^{N-1} D g\left(X_{t_{i}}\right) f\left(X_{t_{i}}\right) B_{t_{i}, t_{i+1}}+ \\
+\sum_{i=0}^{N-1}\left(D g\left(X_{t_{i}}\right) D f\left(X_{t_{i}}\right) f\left(X_{t_{i}}\right) \mathbb{B}_{t_{i}, t_{i+1}}+\frac{1}{2} D^{2} g\left(X_{t_{i}}\right)\left(f\left(X_{t_{i}}\right) B_{t_{i}, t_{i+1}}\right)^{\otimes 2}\right)+ \\
+O\left(\left|\mathcal{P}^{(N)}\right|^{3 H-1}\right)=\sum_{i=0}^{N-1} D g\left(X_{t_{i}}\right) f\left(X_{t_{i}}\right) B_{t_{i}, t_{i+1}}+ \\
+\sum_{i=0}^{N-1}\left(D g\left(X_{t_{i}}\right) D f\left(X_{t_{i}}\right) f\left(X_{t_{i}}\right) \mathbb{B}_{t_{i}, t_{i+1}}+f\left(X_{t_{i}}\right)^{\mathrm{T}} D^{2} g\left(X_{t_{i}}\right) f\left(X_{t_{i}}\right) \mathbb{B}_{t_{i}, t_{i+1}}\right)+ \\
+\sum_{i=0}^{N-1} f\left(X_{t_{i}}\right)^{\mathrm{T}} D^{2} g\left(X_{t_{i}}\right) f\left(X_{t_{i}}\right)\left(\frac{1}{2}\left(B_{t_{i}, t_{i+1}}\right)^{\otimes 2}-\mathbb{B}_{t_{i}, t_{i+1}}\right)+O\left(\left|\mathcal{P}^{(N)}\right|^{3 H-1}\right) .
\end{gathered}
$$

Обозначим $\widetilde{\mathbb{B}}_{s, t}=\mathbb{B}_{s, t}+\frac{1}{2}(t-s) I_{\bar{H}}$. Так как пара $(B, \widetilde{\mathbb{B}})$ принадлежит пространству геометрических грубых траекторий, то $\operatorname{Sym}\left(\widetilde{\mathbb{B}}_{t_{i}, t_{i+1}}\right)=\frac{1}{2}\left(B_{t_{i}, t_{i+1}}\right)^{\otimes 2} \quad$ и $\quad \frac{1}{2}\left(B_{t_{i}, t_{i+1}}\right)^{\otimes 2}-\mathbb{B}_{t_{i}, t_{i+1}}=$ $=-\operatorname{Anti}\left(\widetilde{\mathbb{B}}_{i}, t_{i+1}\right)+\frac{1}{2}\left(t_{i+1}-t_{i}\right) I_{\bar{H}}$, где $\operatorname{Anti}(\widetilde{\mathbb{B}})=\frac{1}{2}\left(\widetilde{\mathbb{B}}-\widetilde{\mathbb{B}}^{\mathrm{T}}\right)-$ антисимметричная часть $\widetilde{\mathbb{B}}$. Заметим, что $f(X .)^{\mathrm{T}} D^{2} g(X) f.(X$.$) симметрично, в то время как \operatorname{Anti}(\widetilde{\mathbb{B}})$ антисимметрично, поэтому $f\left(X_{t_{i}}\right)^{\mathrm{T}} D^{2} g\left(X_{t_{i}}\right) f\left(X_{t_{i}}\right) \operatorname{Anti}\left(\widetilde{\mathbb{B}}_{t_{i}, t_{i+1}}\right)$ зануляется для каждого $i=0, \ldots, N-1$. Учитывая этот факт и соотношения

$$
\begin{gathered}
(D g(X .) \cdot f(X .))^{\prime}=D(D g \cdot f)(X .) \cdot X^{\prime}= \\
=f(X .)^{\mathrm{T}} D^{2} g(X .) f(X .)+D g(X .) D f(X .) f(X .), \\
f(X .)^{\mathrm{T}} D^{2} g(X .) f(X .) I_{\bar{H}}=\operatorname{tr}\left(\left(f(X .) I_{\bar{H}}\right)^{T} D^{2} g(X .)\left(f(X .) I_{\bar{H}}\right)\right),
\end{gathered}
$$

из равенства (9) получим 


$$
\begin{aligned}
& g\left(X_{t}\right)-g\left(X_{s}\right)=\sum_{i=0}^{N-1}\left(D g\left(X_{t_{i}}\right) f\left(X_{t_{i}}\right) B_{t_{i}, t_{i+1}}+(D g(X .) f(X .))_{t_{i}}^{\prime} \mathbb{B}_{t_{i}, t_{i+1}}\right)+ \\
& +\sum_{i=0}^{N-1} \frac{1}{2} \operatorname{tr}\left(\left(f\left(X_{t_{i}}\right) I_{\bar{H}}\right)^{T} D^{2} g\left(X_{t_{i}}\right)\left(f\left(X_{t_{i}}\right) I_{\bar{H}}\right)\right)\left(t_{i+1}-t_{i}\right)+O\left(\left|\mathcal{P}^{(N)}\right|^{3 H-1}\right) .
\end{aligned}
$$

Переходя к пределу в равенстве (10) при $\left|\mathcal{P}^{(N)}\right| \rightarrow 0$, получим требуемую формулу замены переменных. Теорема доказана.

Положим $B_{t}^{(d+1)}=t$ и будем считать, что интегралы по $B_{t}^{(d+1)}$ понимаются как интегралы Лебега.

Введем следующие обозначения:

$$
\begin{gathered}
\Delta^{k}[0, t]=\left\{\left(t_{1}, \ldots, t_{k}\right) \in[0,1]^{k}: 0 \leq t_{1}<\ldots<t_{k} \leq t\right\}, \\
\int_{\Delta^{k}[0, t]} d B^{\left(I_{k}\right)}=\int_{0}^{t} \int_{0}^{t_{k}} \ldots \int_{0}^{t_{2}} d B_{t_{1}}^{\left(i_{1}\right)} \ldots d B_{t_{k-1}}^{\left(i_{k-1}\right)} d B_{t_{k}}^{\left(i_{k}\right)}, \\
I_{k}=\left(i_{1}, \ldots, i_{k}\right) \in \mathbb{N}_{d+1}^{k}:=\{0, \ldots, d+1\}^{k}, \\
D_{f}^{(i)}=\sum_{j=1}^{n} f_{j i}(x) \frac{\partial}{\partial x_{j}}, \quad i \in\{0, \ldots, d\}, \\
D_{f}^{(d+1)}=\frac{1}{2} \sum_{k=0}^{d} 1_{\left\{H_{k}=1 / 2\right\}} \sum_{i, j=1}^{n} f_{i k}(\cdot) f_{j k}(\cdot) \frac{\partial^{2}}{\partial x_{i} \partial x_{j}}, \\
D_{f}^{\left(I_{k}\right)}=D_{f}^{\left(i_{1}\right)} \ldots D_{f}^{\left(i_{k}\right)}, \\
P_{t} g(x)=\mathbb{E}_{g} g\left(X_{t}^{x}\right), \quad t \geq 0 .
\end{gathered}
$$

В дальнейшем для краткости будем опускать верхний индекс $x$ в обозначении решения $X_{t}^{x}$ уравнения (2) с начальным условием $X_{0}=x$.

Те орема 4. Пусть $f \in C_{b}^{2 N+2}\left(\mathbb{R}^{n}, \mathbb{R}^{n \times(d+1)}\right), g \in C_{b}^{2 N+3}\left(\mathbb{R}^{n}, \mathbb{R}\right), N \in \mathbb{N}$. Тогда для любого фиксированного $H \in(1 / 3,1 / 2]$ такого, что $H<H_{\min }=\min _{i=0, \ldots d} H_{i}$ справедливо следующее асимптотическое разложение:

$$
P_{t} g(x)=g(x)+\sum_{k=1}^{N} \sum_{I_{k} \in \mathbb{N}_{d+1}^{k}} t^{\left|H_{I_{k}}\right|} \cdot\left(D_{f}^{\left(I_{k}\right)} g\right)(x) \mathbb{E}\left(\int_{\Delta^{k}[0,1]} d B^{\left(I_{k}\right)}\right)+O\left(t^{(N+1) H}\right),
$$

при $t \rightarrow 0$, где $\left|H_{I_{k}}\right|=H_{i_{1}}+H_{i_{2}}+\ldots+H_{i_{k}}-$ сумма индексов Херста дробных броуновских движений $B^{\left(i_{1}\right)}, B^{\left(i_{2}\right)}, \ldots, B^{\left(i_{k}\right)}$.

Док а з а т ел ь с т в о. С учетом введенных обозначений формулу замены переменных можно записать в следующей форме:

$$
g\left(X_{t}^{x}\right)=g(x)+\sum_{i=0}^{d+1} \int_{0}^{t}\left(D_{f}^{(i)} g\right)\left(X_{r}^{x}\right) d B_{r}^{(i)} .
$$

Применяя формулу (12) $(N+1)$ раз, получим

$$
\begin{gathered}
g\left(X_{t}\right)=g(x)+\sum_{k=1}^{N} \sum_{I_{k} \in \mathbb{N}_{d+1}^{k}}\left(D_{f}^{\left(I_{k}\right)} g\right)(x) \int_{\Delta^{k}[0, t]} d B^{\left(I_{k}\right)}+ \\
+\sum_{I_{N+1} \in \mathbb{N}_{d+1}^{N+1}} \int_{0}^{t} \int_{0}^{t_{N+1}} \ldots \int_{0}^{t_{2}}\left(D_{f}^{\left(I_{N+1}\right)} g\right)\left(X_{t_{1}}\right) d B_{t_{1}}^{\left(i_{1}\right)} \ldots d B_{t_{N}}^{\left(i_{N}\right)} d B_{t_{N+1}}^{\left(i_{N+1}\right)} .
\end{gathered}
$$
процесс

Обозначим $\varphi_{I_{N+1}}(x)=\left(D_{f}^{\left(I_{N+1}\right)} g\right)(x)$ и преобразуем последнее слагаемое в (13). Определим 


$$
\widehat{B}_{u}^{(c)}=\left(\widehat{B}_{u}^{(0 ; c)}, \hat{B}_{u}^{(1 ; c)}, \ldots, \hat{B}_{u}^{(d+1 ; c)}\right)^{T}
$$

зависящий от параметра $c>0, i$-я компонента которого определяется равенством $\widehat{B}_{u}^{(i ; c)}=c^{H_{i}} B_{u / c}^{(i)}$, $u \in[0, T]$. По свойству самоподобия дробного броуновского движения процесс $\hat{B}_{u}^{(i ; c)}$ также является дробным броуновским движением с индексом Херста $H_{i}$ для любого $c>0, i=\overline{1, d}$. Следовательно, при фиксированном $t \in[0, T]$ имеем

$$
\begin{aligned}
& \int_{0}^{t} \int_{0}^{t_{N+1}} \ldots \int_{0}^{t_{2}} \varphi_{I_{N+1}}\left(X_{t_{1}}\right) d B_{t_{1}}^{\left(i_{1}\right)} \ldots d B_{t_{N}}^{\left(i_{N}\right)} d B_{t_{N+1}}^{\left(i_{N+1}\right)}= \\
& =\int_{0}^{1} d B_{t \cdot t_{N+1}}^{\left(i_{N+1}\right)} \int_{0}^{t_{N+1}} d B_{t \cdot t_{N}}^{\left(i_{N}\right)} \cdots \int_{0}^{t_{2}} \varphi_{I_{N+1}}\left(X_{t \cdot t_{1}}\right) d B_{t \cdot t_{1}}^{\left(i_{1}\right)}= \\
& \left.\stackrel{\mathcal{L}}{=} \int_{0}^{1} d \widehat{B}_{t \cdot t_{N+1}}^{\left(i_{N+1} ; t\right)} \int_{0}^{t_{N+1}} d \widehat{B}_{t \cdot t_{N}}^{\left(i_{N} ; t\right)} \ldots \int_{0}^{t_{2}} \varphi_{I_{N+1}}\left(\widehat{X}_{t \cdot t_{1}}^{(t)}\right)\right) d \widehat{B}_{t \cdot t_{1}}^{\left(i_{1} ; t\right)}= \\
& =t^{H_{i_{1}}+\ldots+H_{i_{N+1}}} \int_{0}^{1} d B_{t_{N+1}}^{\left(i_{N+1}\right)} \int_{0}^{t_{N+1}} d B_{t_{N}}^{\left(i_{N}\right)} \ldots \int_{0}^{t_{2}} \varphi_{I_{N+1}}\left(\widehat{X}_{t \cdot t_{1}}^{(t)}\right) d B_{t_{1}}^{\left(i_{1}\right)},
\end{aligned}
$$

где знак $\stackrel{\mathcal{L}}{=}$ означает совпадение распределений, а $\widehat{X}_{\tau}^{(t)}$ - решение уравнения

$$
d \widehat{X}_{\tau}^{(t)}=f\left(\widehat{X}_{\tau}^{(t)}\right) d \widehat{B}_{\tau}^{(t)}, \quad \tau \in[0, T]
$$

с начальным условием $\widehat{X}_{0}^{(t)}=x$. Аналогично

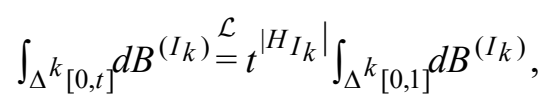

а следовательно, из (13)-(16) получим

$$
P_{t} g(x)=g(x)+\sum_{k=1}^{N} \sum_{I_{k} \in \mathbb{N}_{d+1}^{k}} t^{\left|H_{I_{k}}\right|}\left(D_{f}^{\left(I_{k}\right)} g\right)(x) \mathbb{E}\left(\int_{\Delta^{k}[0,1]} d B^{\left(I_{k}\right)}\right)+\mathcal{R}_{N+1}(t),
$$

где

$$
\begin{gathered}
\mathcal{R}_{N+1}(t)= \\
=\sum_{I_{N+1} \in \mathbb{N}_{d+1}^{N+1}}\left(t^{H_{I_{N+1}} \mid} \mathbb{E} \int_{0}^{1} \int_{0}^{t_{N+1}} \ldots \int_{0}^{t_{2}}\left(D_{f}^{\left(I_{N+1}\right)} g\right)\left(\widehat{X}_{t \cdot t_{1}}^{(t)}\right) d B_{t_{1}}^{\left(i_{1}\right)} \ldots d B_{t_{N}}^{\left(i_{N}\right)} d B_{t_{N+1}}^{\left(i_{N+1}\right)}\right) .
\end{gathered}
$$

Поскольку $\left|H_{I_{N+1}}\right| \geq(N+1) H$ для любого $I_{N+1}$, то при $t<1$ имеем

$$
\begin{gathered}
\left|\mathcal{R}_{N+1}(t)\right| \leq(d+1)^{N+1} t^{(N+1) H} \times \\
\times \max _{I_{N+1} \in \mathbb{N}_{d+1}^{N+1}} \mathbb{E}\left|\int_{0}^{1} \int_{0}^{t_{N+1}} \ldots \int_{0}^{t_{2}}\left(D_{f}^{\left(I_{N+1}\right)} g\right)\left(\widehat{X}_{t \cdot t_{1}}^{(t)}\right) d B_{t_{1}}^{\left(i_{1}\right)} \ldots d B_{t_{N}}^{\left(i_{N}\right)} d B_{t_{N+1}}^{\left(i_{N+1}\right)}\right| .
\end{gathered}
$$

Поэтому, учитывая (17)-(19), для завершения доказательства формулы (11) осталось показать, что

$$
\mathbb{E}\left|\int_{0}^{1} \int_{0}^{t_{N+1}} \ldots \int_{0}^{t_{2}}\left(D_{f}^{\left(I_{N+1}\right)} g\right)\left(\widehat{X}_{t \cdot t_{1}}^{(t)}\right) d B_{t_{1}}^{\left(i_{1}\right)} \ldots d B_{t_{N}}^{\left(i_{N}\right)} d B_{t_{N+1}}^{\left(i_{N+1}\right)}\right|<+\infty
$$

для любых $I_{N+1}=\left(i_{1}, \ldots, i_{N+1}\right) \in \mathbb{N}_{d+1}^{N+1}$. 
Соотношение (20) вытекает из предложения 1 и леммы 7.5 из [18]. Теорема доказана.

Далее рассматриваем уравнение (1)

$$
d X_{t}=b\left(X_{t}\right) d t+\sigma\left(X_{t}\right) d B_{t}+h\left(X_{t}\right) d W_{t}, \quad t \in[0, T] .
$$

Не нарушая общности, будем считать, что процессы $X_{t}, W_{t}, B_{t}$ принимают значения в $d$-мерном евклидовом пространстве. Будем предполагать, что функции $b, h, \sigma$ имеют непрерывные

и ограниченные частные производные до второго порядка включительно. Положим $\tilde{b}=b-\frac{1}{2} h_{x}^{\prime} h$.
Рассмотрим автономное обыкновенное дифференциальное уравнение

$$
d z_{t}=G\left(z_{t}\right) d t, \quad t \in \mathbb{R},
$$

где $G=\operatorname{col}\left(G^{1}, \ldots, G^{d}\right) \in C_{b}^{2}\left(\mathbb{R}^{d}, \mathbb{R}^{d}\right)$, и обозначим через $z_{t}^{y}$ единственное решение данного уравнения с начальным условием $z_{0}=0$.

Нетрудно видеть, что $z_{t}^{y}=T_{G}(t) y$, где $T_{G}(t):=e^{t M_{G}}-C_{0}$-полугруппа на $C\left(\mathbb{R}^{d}, \mathbb{R}^{d}\right)$, порожденная дифференциальным оператором $M_{G}: C^{1}\left(\mathbb{R}^{d}, \mathbb{R}^{d}\right) \rightarrow C\left(\mathbb{R}^{d}, \mathbb{R}^{d}\right)$, действующим по правилу

$$
\left(M_{G} w\right)(y)=\operatorname{col}\left(\sum_{i=1}^{d} G^{i}(y) \frac{\partial w^{1}}{\partial y_{i}}, \ldots, \sum_{i=1}^{d} G^{i}(y) \frac{\partial w^{d}}{\partial y_{i}}\right),
$$

где $w=\operatorname{col}\left(w^{1}, \ldots, w^{d}\right) \in C^{1}\left(\mathbb{R}^{d}, \mathbb{R}^{d}\right), \quad y \in \mathbb{R}^{d}$.

Оп ределе н и 2 . Будем говорить, что семейство отображений $\left\{G_{\alpha}\right\}_{\alpha \in A} \subseteq C_{b}^{2}\left(\mathbb{R}^{d}, \mathbb{R}^{d}\right)$ порождает коммутирующие потоки, если для любых $\alpha, \beta \in A$ операторы $T_{G_{\alpha}}\left(t_{1}\right), T_{G_{\beta}}\left(t_{2}\right)$ перестановочные для любых $t_{1}, t_{2} \in \mathbb{R}$.

Обозначим $\Gamma=\{\tilde{b}\} \cup\left\{h_{j} \mid j=1, \ldots, d\right\} \cup\left\{\sigma_{j} \mid j=1, \ldots, d\right\}$. Через $X_{t}^{y}$ будем обозначать решение уравнения (1) с начальным условием $X_{0}=y \in \mathbb{R}^{d}$.

Определим функцию $F: \mathbb{R}^{3 d+1} \rightarrow \mathbb{R}^{d}$ следующим образом:

$$
F\left(y, t, s_{1}, \ldots, s_{d}, \tau_{1}, \ldots, \tau_{d}\right)=\left(T_{\tilde{b}}(t) T_{h_{1}}\left(s_{1}\right) \ldots T_{h_{d}}\left(s_{d}\right) T_{\sigma_{1}}\left(\tau_{1}\right) \ldots T_{\sigma_{d}}\left(\tau_{d}\right)\right)(y),
$$

где $y \in \mathbb{R}^{d}, t \in \mathbb{R}, s_{j}, \tau_{j} \in \mathbb{R}(j=1, \ldots, d)$.

Пр ед ло же н и е 2. Если семейство Г порождает коммутирующие потоки, то

$$
X_{t}^{y}=F\left(y, t, W_{t}^{(1)}, \ldots, W_{t}^{(d)}, B_{t}^{(1)}, \ldots, B_{t}^{(d)}\right)
$$

n. н. для любого $t \in[0, T]$.

Дока з ате льс т в о. Выберем произвольные $y \in \mathbb{R}, t \in[0, T]$ и зафиксируем их. Применяя формулу Ито к процессу $x^{y}(t)$, при этом используя условие коммутирования операторов $T_{\alpha}\left(t_{1}\right), T_{\beta}\left(t_{2}\right), \alpha, \beta \in \Gamma, t_{1}, t_{2} \in \mathbb{R}$, получим соотношения

$$
\begin{gathered}
F\left(y, t, W_{t}^{(1)}, \ldots, W_{t}^{(d)}, B_{t}^{(1)}, \ldots, B_{t}^{(d)}\right)= \\
=F(y, 0, \ldots, 0)+\int_{0}^{t}\left(\frac{\partial F\left(y, u, W_{u}, B_{u}\right)}{\partial t}+\frac{1}{2} \sum_{j=1}^{d} \frac{\partial^{2} F\left(y, u, W_{u}, B_{u}\right)}{\partial s_{j}^{2}}\right) d u+ \\
+\sum_{j=1}^{d} \int_{0}^{t} \frac{\partial F\left(y, u, W_{u}, B_{u}\right)}{\partial s_{j}} d W_{u}^{j}+\sum_{j=1}^{d} \int_{0}^{t} \frac{\partial F\left(y, u, W_{u}, B_{u}\right)}{\partial \tau_{j}} d B_{u}^{j}= \\
=y+\int_{0}^{t} b\left(F\left(y, u, W_{u}, B_{u}\right)\right) d u+\sum_{j=1}^{d} \int_{0}^{t} h_{j}\left(F\left(y, u, W_{u}, B_{u}\right)\right) d W_{u}^{j}+\sum_{j=1}^{d} \int_{0}^{t} \sigma_{j}\left(F\left(y, u, W_{u}, B_{u}\right)\right) d B_{u}^{j} .
\end{gathered}
$$


Непрерывность по Гельдеру траекторий процесса $X_{t}^{y}$ вытекает из непрерывной дифференцируемости функции $F$ и непрерывности по Гельдеру с любым показателем $\alpha<\min _{j} H_{j}$ траекторий процесса $B_{t}$, а также непрерывности по Гельдеру с любым показателем $\alpha<1 / 2$ траекторий процесса $W_{t}$. Предложение доказано.

Те о ре ма 5. Пусть семейство Г порождает коммутирующие потоки, функиия $g: \mathbb{R}^{d} \rightarrow \mathbb{R}$ вместе со своими частными производными до второго порядка включительно непрерывна и ограничена. Тогда функция $u_{g}(y, t)=\mathbb{E}\left(g\left(X_{t}^{y}\right)\right)$ удовлетворяет уравнению

$$
\begin{gathered}
\frac{\partial u_{g}(y, t)}{\partial t}=\sum_{j=1}^{d} b_{j}(y) \frac{\partial u_{g}(y, t)}{\partial y_{j}}+\frac{1}{2} \sum_{i, j, k=1}^{d} h_{i k}(y) h_{j k}(y) \frac{\partial^{2} u_{g}(y, t)}{\partial y_{i} \partial y_{j}}+ \\
+\sum_{i, j, k=1}^{d} H_{j} t^{2 H_{j}-1} \sigma_{i k}(y)\left(\sigma_{j k}(y) \frac{\partial^{2} u_{g}(y, t)}{\partial y_{i} \partial y_{j}}+\frac{\partial \sigma_{j k}(y)}{\partial y_{i}} \frac{\partial u_{g}(y, t)}{\partial y_{j}}\right), \quad t>0, \quad y \in \mathbb{R}^{d},
\end{gathered}
$$

и начальному условию $u_{g}(y, 0)=g(y), y \in \mathbb{R}^{d}$.

Док аз а т ел ь с т в о. Определим функцию $G_{d}: \mathbb{R}^{d+1} \rightarrow \mathbb{R}$ следующим образом:

$$
G_{d}\left(y, \tau_{d}\right)=g\left(T_{\sigma_{d}}\left(\tau_{d}\right)(y)\right), \quad y \in \mathbb{R}^{d}, \quad \tau_{d} \in \mathbb{R} .
$$

Применяя формулу Ито к процессу $G_{d}\left(y, B_{t}^{(d)}\right)$, получим соотношение

$$
G_{d}\left(y, B_{t}^{(d)}\right)=G_{d}(y, 0)+\int_{0}^{t} \frac{\partial G_{d}\left(y, B_{s}^{(d)}\right)}{\partial \tau_{d}} \diamond d B_{s}^{(d)}+\int_{0}^{t} H_{d} s^{2 H_{d}-1} \frac{\partial^{2} G_{d}\left(y, B_{s}^{(d)}\right)}{\partial \tau_{d}^{2}} d s,
$$

где стохастический интеграл в правой части соотношения (21) - интеграл Вика - Ито Скорохода.

Как показано в [17], справедливо равенство

$$
\frac{\partial^{2} G_{d}\left(y, \tau_{d}\right)}{\partial \tau_{d}^{2}}=\left(M_{\sigma_{d}}^{2} G_{d}\left(\cdot, \tau_{d}\right)\right)(y)
$$

Обозначим $\psi_{d}(y, t)=\mathbb{E} G_{d}\left(y, B_{t}^{(d)}\right)$. Тогда из соотношений (21), (22), теоремы Фубини и правила Лейбница вытекает равенство

$$
\psi_{d}(y, t)=\psi_{d}(y, 0)+\int_{0}^{t} H_{d} s^{2 H_{d}-1}\left(M_{\sigma_{d}}^{2} \psi_{d}(\cdot, s)\right)(y) d s .
$$

Из соотношения (23) вытекает справедливость соотношения

$$
\frac{\partial \psi_{d}(\cdot, t)}{\partial t}=H_{d} t^{2 H_{d}-1} M_{\sigma_{d}}^{2} \psi_{d}(\cdot, t) .
$$

Определим функцию $G_{d-1}: \mathbb{R}^{d+1} \times[0, T] \rightarrow \mathbb{R}$ равенством

$$
G_{d-1}\left(y, \tau_{d-1}, t\right)=\psi_{d}\left(T_{\sigma_{d-1}}\left(\tau_{d-1}\right)(y), t\right), \quad y \in \mathbb{R}^{d}, \quad \tau_{d-1} \in \mathbb{R}, \quad t \in[0, T] .
$$

Применяя формулу Ито к процессу $G_{d-1}\left(y, B_{t}^{(d-1)}, t\right)$, получим соотношение

$$
\begin{aligned}
& G_{d-1}\left(y, B_{t}^{(d-1)}, t\right)=G_{d-1}(y, 0,0)+\int_{0}^{t} \frac{\partial G_{d-1}\left(y, B_{s}^{(d-1)}, s\right)}{\partial \tau_{d-1}} \diamond d B_{s}^{(d-1)}+ \\
& +\int_{0}^{t}\left(\frac{\partial G_{d-1}\left(y, B_{s}^{(d-1)}, s\right)}{\partial \tau_{d}}+H_{d-1} s^{2 H_{d-1}-1} \frac{\partial^{2} G_{d-1}\left(y, B_{s}^{(d-1)}, s\right)}{\partial \tau_{d-1}^{2}}\right) d s .
\end{aligned}
$$


Отметим, что для каждых $y \in \mathbb{R}^{d}, \tau_{d-1} \in \mathbb{R}, s \in[0, T]$ справедливо равенство

$$
\frac{\partial^{2} G_{d-1}\left(y, \tau_{d-1}, s\right)}{\partial \tau_{d-1}^{2}}=\left(M_{\sigma_{d-1}}^{2} G_{d-1}\left(\cdot, \tau_{d-1}, s\right)\right)(y) .
$$

Обозначим $\psi_{d-1}(y, t)=\mathbb{E}_{d-1}\left(y, B_{t}^{(d-1)}, t\right)$, тогда с помощью соотношений (24), (25), (26), теоремы Фубини и правила Лейбница, получаем равенство

$$
\psi_{d-1}(y, t)=\psi_{d-1}(y, 0)+\int_{0}^{t}\left(\left(H_{d} s^{2 H_{d}-1} M_{\sigma_{d}}^{2}+H_{d-1} s^{2 H_{d-1}-1_{1}} M_{\sigma_{d-1}}^{2}\right) \psi_{d-1}\right)(\cdot, s)(y) d s .
$$

Из соотношения (27) получаем, что

$$
\frac{\partial \psi_{d-1}(\cdot, t)}{\partial t}=\left(H_{d} t^{2 H_{d}-1} M_{\sigma_{d}}^{2}+H_{d-1} t^{2 H_{d-1}-1} M_{\sigma_{d-1}}^{2}\right) \psi_{d-1}(\cdot, t) .
$$

Далее рассматриваем функцию $G_{d-2}\left(y, \tau_{d-2}, t\right)=\psi_{d-1}\left(T_{b_{d-2}}\left(\tau_{d-2}\right)(y), t\right)$, применяем формулу Ито к процессу $G_{d-2}\left(y, B_{t}^{(d-2)}, t\right)$, получим уравнение, аналогичное уравнению (28) для функции $\psi_{d-2}(y, t)=\mathbb{E} G_{d-2}\left(y, \tau_{d-2}, t\right)$, и т. д. Тем самым придем к следующему уравнению для функции $u_{g}(y, t)=\mathbb{E} g\left(F\left(y, t, W_{t}^{(1)}, \ldots, W_{t}^{(d)}, B_{t}^{(1)}, \ldots, B_{t}^{(d)}\right)\right)$ :

$$
\frac{\partial u_{g}(\cdot, t)}{\partial t}=\left(M_{\tilde{b}}+\frac{1}{2} \sum_{j=1}^{d} M_{h_{j}}^{2}+\sum_{j=1}^{d} H_{j} t^{2 H_{j}-1} M_{\sigma_{j}}^{2}\right) u_{g}(\cdot, t) .
$$

Теорема доказана.

Из теоремы 5 настоящей работы и доказательства теоремы 4 из [17] вытекает следующее утверждение.

С ле д с т в и е 1. Пусть семейство Г порождает коммутирующие потоки, решение $x_{y}(t)$ автономного уравнения (1) с начальным условием $x(0)=y$ имеет плотность распределения $p(t, y, z)$, функиии $f(z), g(z), \sigma(z), p(t, y, z)$ являются достаточно гладкими и ограниченными. Тогда функция $p(t, y, z)$ удовлетворяет равенству

$$
\begin{gathered}
\frac{\partial p(t, y, z)}{\partial t}=-\sum_{j=1}^{d} \frac{\partial\left(b_{j}(z) p(t, y, z)\right)}{\partial z_{j}}+\frac{1}{2} \sum_{i, j, k=1}^{d} \frac{\partial^{2}\left(h_{i k}(z) h_{j k}(z) p(t, y, z)\right)}{\partial z_{i} \partial z_{j}}+ \\
+\sum_{i, j, k=1}^{d} H_{j} t^{2 H_{j}-1}\left(\frac{\partial^{2}\left(\sigma_{i k}(z) \sigma_{j k}(z) p(t, y, z)\right)}{\partial z_{i} \partial z_{j}}-\frac{\partial\left(\sigma_{i k}(z) \frac{\partial \sigma_{j k}(z)}{\partial z_{i}} p(t, y, z)\right)}{\partial z_{j}}\right), t>0, y, z \in \mathbb{R}^{d} .
\end{gathered}
$$

Рассмотрим модель DTSM для прогнозирования ожидаемых кредитных потерь на примере ипотечных кредитов США, описанную в $[24,18]$. Через $a, t, v$ будем обозначать соответственно возраст кредита (в месяцах), текущую дату наблюдения и дату открытия кредита. Будем рассматривать вероятности дефолта $P D$ и закрытия кредита $P A$ как функции от $a, t$, а именно:

$$
P D(a, t)=\frac{N_{\mathrm{def}}(a, t)}{N_{\mathrm{act}}(a, t-1)}, \quad P A(a, t)=\frac{N_{\mathrm{attr}}(a, t)}{N_{\mathrm{act}}(a, t-1)},
$$

где $N_{\text {act }}, N_{\text {def }}, N_{\text {attr }}$ - числа активных, дефолтных и закрытых аккаунтов соответственно.

Определим среднюю величину дефолтного баланса:

$$
E A D(a, t)=\frac{B_{\mathrm{def}}(a, t)}{N_{\mathrm{def}}(a, t)},
$$

где $B_{\text {def }}$ - суммарный дефолтный баланс. 
На первом этапе анализа для каждой из переменных $P D, P A, E A D$ вычисляются следующие APC-декомпозиции (Age-Period-Cohort):

$$
\begin{gathered}
\operatorname{logit}(P D(a, t))=F_{P D}(a)+H_{P D}(t)+G_{P D}(v), \\
\operatorname{logit}(P A(a, t))=F_{P A}(a)+H_{P A}(t)+G_{P A}(v), \\
\log (E A D(a, t))=F_{E A D}(a)+H_{E A D}(t)+G_{E A D}(v),
\end{gathered}
$$

где $\log \operatorname{it}(p)=\log \left(\frac{p}{1-p}\right)$ - обратная к логистической функции $g(z)=\frac{1}{1+e^{-z}}, z \in \mathbb{R}$. В декомпозициях переменных $P D, P A, E A D$ функции $F(a)$ являются жизненными циклами, функции $H(t)$ выражают влияние макроэкономических факторов, функции $G(v)$ включают в себя влияние внутренних факторов неэкономического характера.

Второй этап анализа заключается в построении экономических моделей для функций $H(t)$. Экономические модели строятся на основе данных по основным макроэкономическим переменным:

$$
H(t)=\beta_{0}+\sum_{i=1}^{N} \beta_{i} f_{i}(t)+\varepsilon(t)
$$

где $f_{1}(t)(i=1, \ldots, N)$ - некоторый набор макроэкономических факторов; $\beta_{i}$ - искомые коэффициенты; $\varepsilon(t)$ - погрешность.

При моделировании соответствующих переменных функция $H(t)$ заменяется ее экономическим приближением

$$
\hat{H}(t)=\beta_{0}+\sum_{i=1}^{N} \beta_{i} f_{i}(t)
$$

а функция кредитного риска $G(v)$ заменяется индивидуальными характеристиками кредитных аккаунтов - скоринговыми факторами (персональный кредитный рейтинг - FICO Score, процентная ставка по кредиту, наличие текущей задолженности и др.).

Модель DTSM позволяет строить оценку текущих ожидаемых кредитных потерь в предположении, что известна экстраполяция функций $\hat{H}_{P D}(t), \hat{H}_{P A}(t), \hat{H}_{E A D}(t)$ на весь период существования аккаунтов, активных на текущую дату $t_{0}$.

Для нахождения экстраполяций функций $\hat{H}(t)$ общепринятым является следующий метод [24]. Используя ежегодный краткосрочный прогноз по макроэкономическим показателям, выпускаемый Федеральной резервной системой, осуществляется экстраполяция функции $\hat{H}(t)$ на двухлетний период начиная с момента начала прогнозирования $t_{0}$. Для получения дальнейшей экстраполяции на период существования аккаунтов, активных на момент $t_{0}$, используется идея возврата к среднему на основе модели Васичека: искомая экстраполяция функции $\hat{H}(t)$ задается с помощью решения системы уравнений Орнштейна - Уленбека

$$
\begin{gathered}
d x(t)=(\theta(\mu-x(t))+v(t)) d t, \\
d v(t)=-\theta_{1} v(t) d t+\sigma d W(t)+\sigma_{1} d B^{H}(t),
\end{gathered}
$$

где $\mu$ - долгосрочное среднее; $\sigma, \sigma_{1}>0$ - коэффициенты волатильности; $\theta, \theta_{1}$ - некоторые положительные постоянные, $\theta \neq \theta_{1}$, а $W(t)$ и $B^{H}(t)$ - независимые одномерные стандартное броуновское движение и дробное броуновское движение с индексом Херста $H>1 / 3$.

Нетрудно видеть, что процесс $x(t)$, удовлетворяющий данной системе, задается равенством

$$
x(t)=\mu+C_{1} e^{-\theta t}+C_{2} e^{-\theta_{1} t}+\frac{1}{\theta-\theta_{1}} \int_{0}^{t}\left(e^{-\theta_{1}(t-s)}-e^{-\theta(t-s)}\right) d M(s),
$$


где $M(t)=\sigma W(t)+\sigma_{1} B^{H}(t)$, а интеграл в правой части понимается как потраекторный интеграл Янга. Вычислив математическое ожидание $x(t)$, имеем

$$
\mathbb{E}(x(t))=\mu+C_{1} e^{-\theta t}+C_{2} e^{-\theta_{1} t} \underset{t \rightarrow \infty}{\rightarrow} \mu .
$$

Наилучшей оценкой процесса $x(t)$ является его математическое ожидание $\hat{x}(t)=\mathbb{E}(x(t))$. Отметим, что использование уравнений Орнштейна - Уленбека второго порядка позволяет строить гладкие долгосрочные экстраполяции за счет выбора параметров $C_{1}, C_{2}$ (см. $[24,18]$ ). А использование слагаемых, содержащих дробные броуновские движения, приводит к более точным моделям по сравнению с моделями на основе уравнений Орнштейна - Уленбека, содержащих лишь стандартные броуновские движения.

\section{Список использованных источников}

1. Stochastic Calculus for Fractional Brownian Motion and Applications / F. Biagini [et al.]. - London: Springer-Verlag, 2008. - 330 p. https://doi.org/10.1007/978-1-84628-797-8

2. Cheridito, P. Regularizing fractional Brownian motion with a view towards stock price modeling: a dissertation ... doctor of mathematics / P. Cheridito. - Zurich, 2001. - 121 p.

3. Zahle, M. Integration with respect to fractal functions and stochastic calculus. I / M. Zahle // Probability Theory and Related Fields. - 1998. - Vol. 111, № 3. - P. 333-374. https://doi.org/10.1007/s004400050171

4. Mishura, Y. S. Stochastic calculus for fractional Brownian motion and related processes / Y. S. Mishura. - Berlin; Heidelberg: Springer-Verlag, 2008. - 398 p. https://oi.org/10.1007/978-3-540-75873-0

5. Kleptsyna, M. L. General approach to filtering with fractional Brownian noises application to linear systems / M. L. Kleptsyna, A. Le Breton, M.-C. Roubaud // Stochastics and Stochastic Reports. - 2000. - Vol. 71, № 1/2. - P. $119-140$. https://doi.org/10.1080/17442500008834261

6. Vaskouski, M. Asymptotic expansions of solutions of stochastic differential equations driven by multivariate fractional Brownian motions having Hurst indices greater than 1/3 / M. Vaskouski, I. Kachan // Stochastic Anal. Appl. - 2018. - Vol. 36, № 6. - P. 909-931. https://doi.org/10.1080/07362994.2018.1483247

7. Kubilius, K. The existence and uniqueness of the solution of an integral equation driven by a p-semimartingale of special type / K. Kubilius // Stochastic Processes and their Appl. - 2002. - Vol. 98, № 2. - P. 289-315. https://doi.org/10.1016/ s0304-4149(01)00145-4

8. Guerra, J. Stochastic differential equations driven by fractional Brownian motion and standard Brownian motion / J. Guerra, D. Nualart// Stochastic Anal. Appl.-2008. - Vol. 26, № 5. - P. 1053-1075. https://doi.org/10.1080/07362990802286483

9. Mishura, Y. S. Existence and uniqueness of the solution of stochastic differential equation involving Wiener process and fractional Brownian motion with Hurst index $H>1 / 2$ / Y. S. Mishura, G. M. Shevchenko // Communications in Statistics Theory and Methods. - 2011. - Vol. 40, № 19/20. - P. 3492-3508. https://doi.org/10.1080/03610926.2011.581174

10. Shevchenko, G. M. Mixed stochastic delay differential equations / G. M. Shevchenko // Theory of Probability and Mathematical Statistics. - 2014. - Vol. 89. - P. 181-195. https://doi.org/10.1090/s0094-9000-2015-00944-3

11. Леваков, А. А. Существование слабых решений стохастических дифференциальных уравнений со стандартным и дробным броуновскими движениями и с разрывными коэффициентами / А. А. Леваков, М. М. Васьковский // Дифференц. уравнения. - 2014. - Т. 50, № 2. - С. 187-200.

12. Леваков, А. А. Существование слабых решений стохастических дифференциальных уравнений со стандартным и дробным броуновскими движениями, с разрывными коэффициентами и с частично вырожденным оператором диффузии / А. А. Леваков, М. М. Васьковский // Дифференц. уравнения. - 2014. - Т. 50, № 8. - С. 1060-1076.

13. Васьковский, М. М. Существование слабых решений стохастических дифференциальных уравнений с запаздыванием со стандартным и дробным броуновскими движениями / М. М. Васьковский // Вес. Нац. акад. навук Беларусі. Сер. фіз.-мат. навук. - 2015. - № 1. - С. 22-34.

14. Леваков, А. А. Существование решений стохастических дифференциальных включений со стандартным и дробным броуновскими движениями / А. А. Леваков, М. М. Васьковский // Дифференц. уравнения. - 2015. - Т. 51, № 8. - С. 997-1003.

15. Леваков, А. А. Свойства решений стохастических дифференциальных уравнений со стандартным и дробным броуновскими движениями / А. А. Леваков, М. М. Васьковский // Дифференц. уравнения. - 2016. - Т. 52, № 8. C. 1011-1019.

16. Васьковский, М. М. Устойчивость и притяжение решений нелинейных стохастических дифференциальных уравнений со стандартным и дробным броуновскими движениями / М. М. Васьковский // Дифференц. уравнения. 2017. - Т. 53, № 2. - С. 160-173.

17. Васьковский, М. М. Методы интегрирования стохастических дифференциальных уравнений смешанного типа, управляемых дробными броуновскими движениями / М. М. Васьковский, И. В. Качан // Вес. Нац. акад. навук Беларусі. Сер. фіз.-мат. навук. - 2019. - Т. 55, № 2. - С. 135-151. https://doi.org/10.29235/1561-2430-2019-55-2-135-151 
18. Леваков, А. А. Стохастические дифференциальные уравнения и включения / А. А. Леваков, М. М. Васьковский. - Минск: БГУ, 2019. - 495 с.

19. Lyons, T. Differential equations driven by rough signals / T. Lyons // Revista Matematica Iberoamericana. - 1998. Vol. 14, № 2. - P. 215-310. https://doi.org/10.4171/rmi/240

20. Gubinelli, M. Controlling rough paths / M. Gubinelli // J. Functional Anal. - 2004. - Vol. 216, № 1. - P. 86-140. https://doi.org/10.1016/j.jfa.2004.01.002

21. Friz, P. A Course on Rough Paths with an Introduction to Regularity Structures / P. Friz, M. Hairer. - Cham: Springer Int. Publ. AG, 2014. - 262 p. https://doi.org/10.1007/978-3-319-08332-2

22. Trees and asymptotic expansions for fractional stochastic differential equations / A. Neuenkirch [et al.] // Annales de I Institut Henri Poincaré (B) Probability and Statistics. - 2009. - Vol. 45, № 1. - P. 157-174. https://doi.org/10.1214/07-aihp159

23. Coutin, L. Stochastic analysis, rough path analysis and fractional Brownian motions / L. Coutin, Z. Qian // Probability Theory Related Fields. - 2002. - Vol. 122, № 1. - P. 108-140. https://doi.org/10.1007/s004400100158

24. Breeden, J. L. Living with CECL: Mortgage modeling alternatives / J. L. Breeden. - Middletown, 2018. - 203 p.

\section{References}

1. Biagini F., Hu Y., Oksendal B., Zhang T. Stochastic Calculus for Fractional Brownian Motion and Applications. London, Springer-Verlag, 2008. 330 p. https://doi.org/10.1007/978-1-84628-797-8

2. Cheridito P. Regularizing Fractional Brownian Motion with a View Towards Stock Price Modeling. Zurich, ETH, 2001. $121 \mathrm{p}$.

3. Zahle M. Integration with respect to fractal functions and stochastic calculus. I. Probability Theory and Related Fields, 1998, vol. 111, no. 3, pp. 333-374. https://doi.org/10.1007/s004400050171

4. Mishura Y. S. Stochastic Calculus for Fractional Brownian Motion and Related Processes. Berlin, Heidelberg, Springer-Verlag, 2008. 411 p. https://doi.org/10.1007/978-3-540-75873-0

5. Kleptsyna M. L., Le Breton A., Roubaud M.-C. General approach to filtering with fractional Brownian noises application to linear systems. Stochastics and Stochastic Reports, 2000, vol. 71, no. 1-2, pp. 119-140. https://doi. org/10.1080/17442500008834261

6. Vaskouski M., Kachan I. Asymptotic expansions of solutions of stochastic differential equations driven by multivariate fractional Brownian motions having Hurst indices greater than 1/3. Stochastic Analysis and Applications, 2018, vol. 36, no. 6, pp. 909-931. https://doi.org/10.1080/07362994.2018.1483247

7. Kubilius K. The existence and uniqueness of the solution of an integral equation driven by a p-semimartingale of special type. Stochastic Processes and their Applications, 2002, vol. 98, no. 2, pp. 289-315. https://doi.org/10.1016/s03044149(01)00145-4

8. Guerra J., Nualart D. Stochastic differential equations driven by fractional Brownian motion and standard Brownian motion. Stochastic Analysis and Applications, 2008, vol. 26, no. 5, pp. 1053-1075. https://doi.org/10.1080/07362990802286483

9. Mishura Y. S., Shevchenko G. M. Existence and uniqueness of the solution of stochastic differential equation involving Wiener process and fractional Brownian motion with Hurst index $H>1 / 2$. Communications in Statistics - Theory and Methods, 2011, vol. 40, no. 19-20, pp. 3492-3508. https://doi.org/10.1080/03610926.2011.581174

10. Shevchenko G. M. Mixed stochastic delay differential equations. Theory of Probability and Mathematical Statistics, 2014, vol. 89, pp. 181-195. https://doi.org/10.1090/s0094-9000-2015-00944-3

11. Levakov A. A., Vas'kovskii M. M. Existence of weak solutions of stochastic differential equations with standard and fractional Brownian motions and with discontinuous coefficients. Differential Equations, 2014, vol. 50, no. 2, pp. $189-202$. https://doi.org/10.1134/s0012266114020062

12. Levakov A. A., Vas'kovskii M. M. Existence of weak solutions of stochastic differential equations with standard and fractional Brownian motion, discontinuous coefficients, and a partly degenerate diffusion operator. Differential Equations, 2014, vol. 50, no. 8, pp. 1053-1069. https://doi.org/10.1134/s0012266114080059

13. Vas'kovskii M. M. Existence of weak solutions of stochastic delay differential equations driven by standard and fractional Brownian motions. Vestsi Natsyianal'nai akademii navuk Belarusi. Seryia fizika-matematychnykh navuk = Proceedings of the National Academy of Sciences of Belarus. Physics and Mathematics series, 2015, no. 1, pp. 22-34 (in Russian).

14. Levakov A. A., Vas'kovskii M. M. Existence of solutions of stochastic differential inclusions with standard and fractional Brownian motions. Differential Equations, 2015, vol. 51, no. 8, pp. 991-997. https://oi.org/10.1134/ s0012266115080030

15. Levakov A. A., Vas'kovskii M. M. Properties of solutions of stochastic differential equations with standard and fractional Brownian motions. Differential Equations, 2016, vol. 52, no. 8, pp. 972-980. https://doi.org/10.1134/ s0012266116080024

16. Vas'kovskii M. M. Stability and attraction of solutions of nonlinear stochastic differential equations with standard and fractional Brownian motions. Differential Equations, 2017, vol. 53, no. 2, pp. 157-170. https://doi.org/10.1134/ s0012266117020021

17. Vas'kovskii M. M., Kachan I. V. Integration methods of mixed-type stochastic differential equations with fractional Brownian motions. Vestsi Natsyianal'nai akademii navuk Belarusi. Seryia fizika-matematychnykh navuk = Proceedings of the National Academy of Sciences of Belarus. Physics and Mathematics series, 2019, vol. 55, no. 2, pp. 135-151 (in Russian). https://doi.org/10.29235/1561-2430-2019-55-2-135-151 
18. Levakov A. A., Vas'kovskii M. M. Stochastic Differential Equations and Inclusions. Minsk, BSU, 2019.495 p. (in Russian).

19. Lyons T. Differential equations driven by rough signals. Revista Matematica Iberoamericana, 1998, vol. 14, no. 2 , pp. 215-310. https://doi.org/10.4171/rmi/240

20. Gubinelli M. Controlling rough paths. Journal of Functional Analysis, 2004, vol. 216, no. 1, pp. 86-140. https://doi. org/10.1016/j.jfa.2004.01.002

21. Friz P., Hairer M. A Course on Rough Paths with an Introduction to Regularity Structures. Cham: Springer International Publishing AG, 2014. 262 p. https://doi.org/10.1007/978-3-319-08332-2

22. Neuenkirch A., Nourdin I., Robler A., Tindel S. Trees and asymptotic expansions for fractional stochastic differential equations. Annales de I Institut Henri Poincaré (B) Probability and Statistics, 2009, vol. 45, no. 1, pp. 157-174. https://doi. org/10.1214/07-aihp159

23. Coutin L., Qian Z. Stochastic analysis, rough path analysis and fractional Brownian motions. Probability Theory Related Fields, 2002, vol. 122, no. 1, pp. 108-140. https://doi.org/10.1007/s004400100158

24. Breeden J. L. Living with CECL: Mortgage modeling alternatives. Middletown, 2018. 203 p.

\section{Информация об авторе}

Васьковский Максим Михайлович - кандидат физико-математических наук, доцент, доцент кафедры высшей математики, Белорусский государственный университет (пр. Независимости, 4, 220072, г. Минск, Республика Беларусь). E-mail: vaskovskii@bsu.by. https://orcid. org/0000-0001-5769-3678

\section{Information about the author}

Maksim M. Vas'kovskii - Ph. D. (Physics and Mathematics), Associate Professor of the Department of Higher Mathematics, Belarusian State University (4, Nezavisimosti Ave., 220072, Minsk, Republic of Belarus). E-mail: vaskovskii@bsu.by. https://orcid.org/0000-0001-5769-3678 